\title{
UNA REVISIÓN DE LA INVESTIGACIÓN SOBRE JUSTICIA AMBIENTAL URBANA EN LATINOAMÉRICA
}

\section{A REVIEW OF RESEARCH ON URBAN ENVIRONMENTAL JUSTICE IN LATIN AMERICA}

\author{
José Mario Mayorga Henao ${ }^{1}$ \\ Alexis E. Vásquez²
}

\section{Resumen}

El objetivo de la presente revisión es identificar las tendencias en la investigación sobre justicia ambiental urbana en Latinoamérica. Se consultaron las bases de datos de Scopus, Redalyc, Google Académico y Scielo y se seleccionaron los artículos que cumplieran con los criterios de búsqueda diseñados. Se presenta una descripción bibliométrica de la producción científica sobre justicia ambiental urbana en Latinoamérica y se identifican las principales aproximaciones conceptuales y metodológicas, el tipo de fuentes utilizadas y las principales conclusiones a las que se llegó en cada estudio. Los resultados indican que las investigaciones se han concentrado en ciudades mexicanas y chilenas. Además son recientes, escasas y concentradas en aspectos clásicos de distribución social de males ambientales, como riesgos naturales y contaminación, y de los bienes ambientales, tales como espacios verdes y servicios públicos.

Palabras Clave: Justicia ambiental urbana, revisión, distribución

\begin{abstract}
The main objective to this review is to identify trends in research on urban environmental justice in Latin America. Scopus, Redalyc, Google Academic and Scielo databases were consulted and the articles that met the designed search criteria were selected. A bibliometric description of the scientific production about urban environmental justice in Latin America is presented and the main conceptual and methodological approaches are identified, along with* the type of sources used and the main conclusions reached in each study. The results indicate that the investigations have focused on Mexican and Chilean cities. Additionally, they are also recent, scarce and concentrated in classical aspects of social distribution of environmental damages such as natural hazards and pollution; and environmental goods, such as green spaces and public services.
\end{abstract}

Keywords: Environmental urban justice, review, distribution

\footnotetext{
${ }^{1}$ Sociólogo. Especialista en Estadística. Especialista en Derecho Urbano. Magister en Planeación Urbana, Magister en Geografía. Profesor catedrático. Departamento de Arquitectura. Pontificia Universidad Javeriana, Colômbia. E-mail: jmmayorgahenao@gmail.com

${ }^{2}$ Profesor Asistente. Departamento de Geografía - Universidad de Chile. E-mail: alexvasq@u.uchile.cl
} 


\section{INTRODUCCION}

Como punto de partida es necesario aclarar que se entiende por Justicia Ambiental y su aplicación como marco conceptual a los estudios de la ciudad.

La expresión justicia ambiental emerge en los años setenta, sobre la base de los movimientos sociales que luchaban contra las desigualdades raciales en la distribución de los residuos peligros y las industrias contaminantes en Estados Unidos. Al respecto, los principales referentes en la identificación del fenómeno son Gelobter (1994), Sarokin y Schulkin (1994), Cutter (1995) y Harvey (1996). A continuación se presentaran las principales definiciones empleadas por cada uno de ellos.

Gelobter (1994) entiende que la justicia ambiental implica la acción de reparación o de cambio de las estructuras o situaciones que se derivan de la discriminación ambiental. Para el autor, la discriminación ambiental son las acciones y las prácticas, derivadas de ideologías y estructuras sociales, que preservan y refuerzan los mecanismos de dominación de algunos grupos sobre el medio ambiente, en detrimento de las condiciones de otros grupos subordinados. Entonces la injusticia ambiental se puede entender como un nexo entre tres dimensiones, la injusticia económica, la injusticia social y una incidencia injusta en la calidad del medio ambiente, lo cual produce una opresión sobre las comunidades de grupos étnicos minoritarios y de bajos ingresos.

Por su parte Cutter (1995), define que un medio ambiente sano es un derecho básico de todos los habitantes de la tierra, un derecho reafirmado por la Declaración de Río (ONU, 1992). Sin embargo, afirma que los riesgos ambientales están inequitativamente distribuidos dentro y entre las sociedades, y que estos riesgos afectan a las poblaciones de manera diferente. Por tanto, las desigualdades en la exposición a amenazas, la reducción del riesgo ambiental y la compensación del riesgo ambiental son elementos cruciales en cuestiones de gestión urbana contemporáneas, tanto como los conceptos asociados a la equidad.

Cutter (1995) hace especial énfasis en entender cómo se generan esas inequidades y describir los efectos desproporcionados de la degradación ambiental en las personas y los lugares que habitan. Según sus planteamientos, las condiciones de injusticia ambiental tienen origen en tres fuentes principales de inequidad: sociales, generacionales y procedimentales. Por un lado, las inequidades sociales surgen de factores como la clase social, la raza, el género, el origen étnico y el poder político, los cuales pueden incidir en que exista una exposición desigual 
a las condiciones negativas del medio ambiente. En segundo lugar, se pueden dar condiciones de inequidad en un marco en el que ciertas decisiones o intervenciones puedan afectar de manera diferente a generaciones futuras. Por último, una fuente de inequidad puede ser el grado en el cual las normas y las sanciones por su incumplimiento sean aplicadas de manera diferencial según las características del grupo al que le apliquen (Cutter 1995).

En este contexto, para Cutter (1995) la justicia ambiental es un término con una carga política que denota algún tipo de acción correctiva para mitigar una injusticia impuesta a un grupo específico de personas. Por tanto, la justicia ambiental es la acción política y la movilización social que reúne el compromiso de las entidades públicas y los agentes privados comprometidos en cambiar una situación ambientalmente inequitativa (Cutter 1995).

Por otro lado, Sarokin \& Schulkin (1994) hacen énfasis en que el concepto de Justicia Ambiental surge en el marco de la discusión por la convergencia de los derechos civiles y los derechos ambientales en el ámbito estadunidense. De tal forma, la Justicia Ambiental, como objetivo de lucha de movimientos sociales de base, tiene como meta hacer respetar el cumplimiento de esos derechos de manera equitativa para todos los grupos sociales.

Sarokin \& Schulkin (1994) identifican tres tipos de movimientos sociales que se afilian al concepto de Justicia Ambiental. En primer lugar, están los movimientos que reclaman la existencia de poblaciones que están sometidas a mayor contaminación ambiental y riesgos por la alteración de algunos elementos de la naturaleza (contaminación de ríos, erosión, desmonte de vegetación, etc.). En segundo lugar, se trata de movimientos que luchan porque hay poblaciones que sufren más perjuicios ambientales por su cercanía a infraestructuras contaminantes (plantas de tratamiento, botaderos de basura, fábricas, etc.). Por último, movimientos sociales que proclaman su lucha debido a que hay poblaciones que son excluidas de los procesos de toma de decisión con respecto a su medio ambiente.

Con respecto a la revisión que hace Harvey (1996), el autor identifica que los movimientos de justicia ambiental surgen a partir de las crisis socio ambiental en distintas partes del mundo. Resalta que dichos movimientos definen la desigualdad como elemento central en la determinación de una situación injusta. Al analizar su modo de operación y sus reclamaciones, identifica tres patrones distintivos. El primero es que sospechan de los discursos racionales. El segundo es que rechazan los discursos bio-céntricos a favor de una visión antropocéntrica de la naturaleza. El tercero es que en estos movimientos participa gente que sufre diferentes tipos de marginaciones, ya sea de clase, genero, raza o etnia, juntando así necesidades de justicia ecológica y social. Para Harvey (1996), estas características Ilevan a que 
la lucha por la justicia ambiental se situé en el campo de lo emotivo y lo simbólico (con la ilusión de una edad mítica de la integración orgánica entre hombre y naturaleza), por lo cual adquiere una posición innegociable de intensa moral, intocable por una racionalidad legal o científica.

Como se puede inferir de la revisión anterior, el concepto tiene un origen en la política y en la lucha de distintos movimientos sociales por mejorar las condiciones de su entorno. Sin embargo, el interés que despiertan estos movimientos sociales en la academia lleva a que la justicia ambiental se constituya en un concepto y área de interés para el desarrollo de investigación científica, especialmente en Estados Unidos. Como objeto de investigación, y según Holifield et al (2009) y Walker (2009), se pueden identificar tres dimensiones principales de interés. La primera en surgir y más extendida en la investigación es la dimensión distributiva, importantemente asociada a una aproximación espacial que busca indagar en la asociación espacial entre grados de calidad ambiental diferentes según distintas clasificaciones sociales. La segunda es la dimensión política y de participación, que busca dar cuenta de conflictos ambientales que generan situaciones injustas, mediante la reconstrucción de los discursos de los movimientos sociales y las lógicas de poder subyacentes al conflicto, la cual se puede definir como dimensión participativa y política.

Si bien el concepto de Justicia Ambiental, tanto en la política como en la investigación, se ha utilizado principalmente para dar cuenta de situaciones inequitativas en entornos urbanos, la ciudad como ambiente focal no hace parte esencial de su definición.

La integración del concepto de Justicia Ambiental con la particularidad de la ciudad como formación espacial, social, política y económica, busca superar los límites analíticos que se han puesto entre la actividad humana y el medio ambiente. La investigación social señala que si se quiere comprender una situación injusta con respecto al medio ambiente, este último debe ser definido como el resultado de un proceso en el que están involucrados los individuos, su vida social y su espacialidad construida. Por tanto, significa que la acción transformativa de los seres humanos, especialmente respecto al medio ambiente urbano como una construcción social, debe ser entendida como un proceso ambiental en el que se pueden presentar condiciones inequitativas que muchas veces pueden ser clasificadas como injustas (Harvey 1996).

Por ejemplo, Gelobter (1994) plantea algunos elementos claves que diferencian la lectura de la justicia ambiental urbana de sus contrapartes rurales. Estos elementos se pueden dividir en dos categorías: los que tienen que ver con las condiciones económicas, con especial 
énfasis en la naturaleza y regulación de usos del suelo; y los que tienen que ver con las condiciones históricas y sociales que enfrentan los distintos grupos sociales en la ciudad.

A partir de la comprensión del medio construido en la ciudad como parte del medio ambiente, la reflexión sobre la Justicia Ambiental se abre hacia nuevos horizontes de investigación. La Justicia Ambiental Urbana investiga las implicancias de la distribución de la población en los espacios urbanos, teniendo en cuenta tanto la distribución de los "males ambientales" así como las diferencias en la accesibilidad que tienen distintos grupos sociales a los "bienes ambientales" o amenidades de la ciudad. Por ende, se reflexiona que al igual que los efectos ambientales negativos derivados de las industrias contaminantes o rellenos sanitarios, las funciones ambientales favorables provistas por, por ejemplo, las áreas verdes urbanas igualmente se distribuyen de manera asimétrica en la población. Por tanto, la Justicia Ambiental Urbana no solo se interesa por cómo los impactos ambientales de la contaminación o del riesgo tienen una distribución injusta entre los distintos grupos sociales, sino también de cómo se distribuyen los atributos ambientales positivos entre la población.

En este contexto, la mayoría de estudios sobre Justicia Ambiental Urbana se han centrado en la evaluación de las condiciones de ciudades de Estados Unidos. Allí la mayoría de estudios han encontrado que la población pobre y las minorías raciales y étnicas reciben mayores impactos por la contaminación ambiental y por riesgos tecnológicos (Mohai et al 2009, Jones et al 2014, Zou et al 2014). Además, hay investigaciones en otras parte del norte global, tales como Canada (Dale et al 2015), Australia (Chakraborty \& Green 2014), Francia (Laurian \& Funderburg 2014), Italia (Germani et al 2014) y Portugal (Nogueira et al 2013), en las que se llegan a resultados similares a las ciudades de Estados Unidos. En Europa hay un mayor uso de las condiciones socioeconómicas sobre las étnicas que en Estados Unidos para la identificación de diferentes grupos sociales. Según Walker (2009) la investigación sobre justicia ambiental ha estado centrada muy importantemente en la dimensión distributiva de la justicia ambiental, pero desde hace un par de décadas se ha ido ampliando para incorporar la dimensión de participación y política.

Por su parte, al parecer en Latinoamérica la investigación científica sobre justicia ambiental urbana ha sido escaza y ha comenzado recientemente. Es necesario tener en cuenta que Latinoamérica es la segunda región más urbanizada en el mundo, con cerca del $80 \%$ de la población habitando en ciudades (Naciones Unidas 2014). El proceso de urbanización se dio de manera explosiva entre los años 1950s y 1960s con las intensas migraciones rurales - urbanas, y tuvo como consecuencia un rápido y descontrolado crecimiento de las áreas urbanas en la 
región. En el contexto de las políticas liberales de sustitución de importaciones y el incipiente proceso de industrialización, millones de habitantes de las áreas rurales migraron a las ciudades en busca de nuevas oportunidades. La llegada de altos flujos migratorios en un corto periodo de tiempo excedió la capacidad de las economías urbanas para absorber la fuerza de trabajo y la de los gobiernos nacionales y locales para satisfacer las demandas de vivienda y de equipamientos urbanos básicos (Romero 2007). Como consecuencia, en Latinoamérica los nuevos pobladores pobres de las ciudades tuvieron que ocupar áreas periféricas, sin dotaciones urbanas y en muchas ocasiones expuestas a riesgos naturales (deslizamiento, inundaciones, etc.) o antrópicos (contaminación industrial, minería, etc.) (Jiménez 2015).

De acuerdo al Atlas de Justicia Ambiental (Atlas de Justicia Ambiental, 2016), los conflictos más importantes en la región se deben a el manejo de residuos sólidos y basuras, el acceso al agua y los impactos de la contaminación industrial. Como ejemplo de lo primero, está el caso de los movimientos sociales que se oponen al relleno sanitario de "Doña Juana" en Bogotá-Colombia y el botadero a cielo abierto manejado por la empresa "Tersa del Golfo" en La Provincia de México- México. En segundo lugar, existen movimientos sociales que debaten sobre la gestión y acceso equitativo al agua, los cuales entran en conflicto con proyectos como la hidroeléctrica "Alto Maipo" en Santiago de Chile, el funcionamiento de la hidroeléctrica de Sogamoso en Colombia o la privatización del agua en la ciudad de Guayaquil en Ecuador. Por último, se ha gestado activismo en torno a los impactos que generan las industrias en la calidad de vida de la población, como la lucha de las comunidades cercanas a industrias que usan asbesto en Bogotá-Colombia o la lucha contra la producción de cemento en las comunidades de Hidalgo-México.

Sin embargo, mientras el activísimo social y político sobre justicia ambiental se ha consolidado en los últimos años en la región, el estado de la investigación en justicia ambiental urbana es menos conocido y, por lo tanto, no resulta fácil identificar las necesidades de investigación y como estas se articulan con la agenda general de justicia ambiental urbana en la región (Vásquez et al 2017). De esta forma, el presente artículo realiza una revisión del estado de la investigación en la región analizando las tendencias de producción científica en los países de la región. El análisis bibliográfico hace énfasis en la identificación de principales conceptos y tipos de metodología, incluyendo técnicas de investigación, fuentes de datos e indicadores en la investigación social sobre justicia ambiental urbana en Latinoamérica. 


\section{MATERIALES Y MÉTODO}

Se realizó una revisión sistemática de literatura (Khan et al 2003) por medio de una búsqueda bibliográfica en las siguientes bases de datos: Scopus, Redalyc, Scielo, Latindex y Google Académico. Estas se seleccionaron debido a su cobertura de revistas Latinoamericanas. Los términos de búsqueda y operadores booleanos que se emplearon son los siguientes: (i) Justicia ambiental AND Urbana, (ii) Justicia ambiental AND Ciudad, (iii) Environmental justice AND Urban, (iv) Environmental justice AND City.

En la búsqueda se utilizó como filtro el área de conocimiento, dejando solo resultados validos en las publicaciones adscritas a las Ciencias Sociales y a las Ciencias ambientales. Este procedimiento dio como resultado 420 publicaciones en Scopus, 9 publicaciones en Google Académico, dos publicaciones en Scielo y una publicación en Redalyc. Los títulos y resúmenes de cada artículo fueron revisados respecto a su relevancia para el tema de la justicia ambiental urbana y su referencia a uno o varios países latinoamericanos.

Como resultado de lo anterior 396 artículos fueron removidos de la base de datos y 24 (4.7\% del total de publicaciones a nivel mundial) publicaciones fueron seleccionadas para su análisis en profundidad. El primer paso del análisis consistió en sistematizar la información usando una matriz que permitiera comparar la información básica de los artículos, así como sus metodologías y principales conclusiones. La matriz diseñada contiene (1) información básica del artículo tal como título, año de publicación, autores e indización, y (2) información sobre una lista de criterios detallados en la Tabla 1.

\section{Tabla 1: Criterios de análisis de los artículos seleccionados}

\begin{tabular}{|c|c|}
\hline Criterio de evaluación & Posible entrada \\
\hline Tipo de artículo & De corte empírico o teórico. \\
\hline Tipo de metodología & $\begin{array}{c}\text { Cualitativa, cuantitativa o una combinación de ambas. } \\
\text { Se incluye un análisis de las fuentes y tipos de datos así } \\
\text { como de los procedimientos de análisis. }\end{array}$ \\
\hline Temas de investigación & $\begin{array}{c}\text { Contaminación, riesgos, acceso a espacios verdes y } \\
\text { conflictos socio ambientales, entre otros. }\end{array}$ \\
\hline $\begin{array}{c}\text { Dimensión de justicia } \\
\text { ambiental estudiada }\end{array}$ & $\begin{array}{c}\text { Distributiva, participación y política, procedimental o } \\
\text { más de una }\end{array}$ \\
\hline Principales resultados & Principales hallazgos de la investigación \\
\hline
\end{tabular}


La información básica permitió calcular métricas respecto, por ejemplo, el idioma en que se publica y los países más productivos. Los resultados de la evaluación en profundidad de los artículos usando la pauta de evaluación permitieron identificar el estado de la investigación en justicia ambiental urbana en Latinoamérica, especialmente respecto los enfoques, los países de aplicación, de los métodos y los resultados de las investigaciones respecto a temas principales y metodologías.

\section{RESULTADOS}

Existen 24 artículos científicos sobre justicia ambiental urbana en Latinoamérica, 12 de ellos que representan el 50\% se encuentran en los registros de Scopus. Nueve artículos fueron localizados en Google Académico, dos en Scielo y uno en Redalyc los cuales representan el 37\%, $2 \%$ y $1 \%$ respectivamente. Es importante destacar que la mayoría de la producción científica sobre Justicia Ambiental Urbana en la región se encuentra en revistas Scopus, que destacan por tener un alto impacto y altos niveles de exigencia en la evaluación de los artículos. Esto se ajusta al interés general de los investigadores de todos los campos en publicar los resultados de sus investigaciones en revistas de alto impacto.

Con respecto a los años de publicación, únicamente se encontraron artículos para el periodo comprendido entre los años 2003 y 2015. Los años de mayor volumen de publicación sobre Justicia Ambiental Urbana en Latinoamérica fueron el 2009 con seis y el 2012 y el 2013 con cuatro publicaciones. Le sigue el año 2015 con tres publicaciones. En los años 2003, 2007, 2008, 2010, 2011 y 2014 solo se identifica una publicación en cada uno. Lo anterior demuestra lo novedoso del tema en la región, dado que es un problema de investigación que despierta el interés de la academia en los últimos 15 años, más sin embargo, en el corto periodo de tiempo no se identifica una tendencia clara de producción.

Fig. $N^{\circ} 1$. Número de artículos según año de publicación 


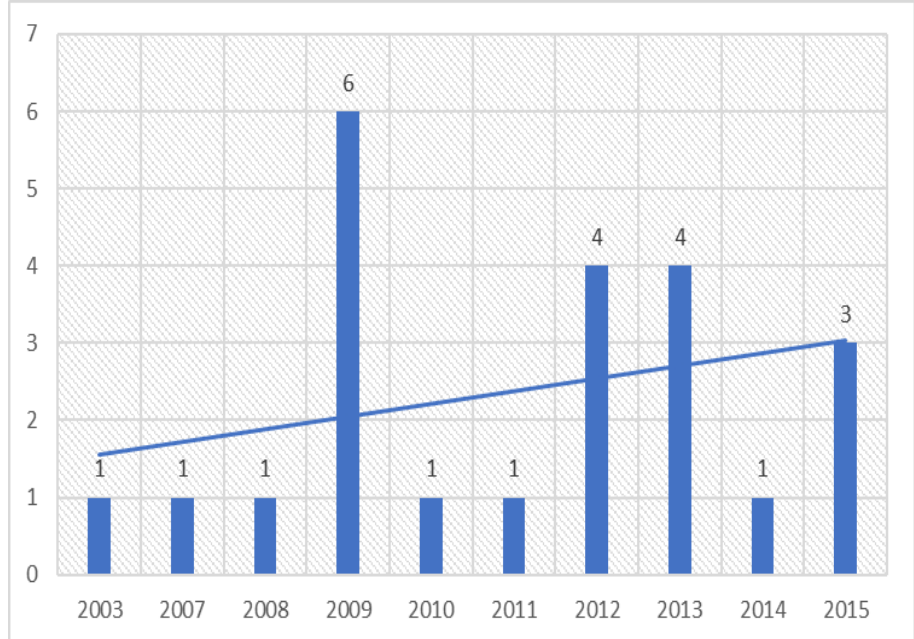

Fuente: Base de datos Scopus, Redalyc y Scielo, Enero de 2017. Elaboración propia.

Existe un predominio del inglés como idioma de publicación de los artículos sobre Justicia Ambiental Urbana en Latinoamérica. El 60\% de los artículos han sido publicados en Ingles, mientras que el $40 \%$ restante han sido publicados en español. Los 13 artículos escritos en inglés han sido publicados en revistas de origen Estadounidense. En revistas propiamente Latinoamericanas se identificaron 10 artículos y uno en revista Española.

\section{PAÍSES SOBRE LOS QUE SE INVESTIGA}

Se pudo identificar que la mayor cantidad de publicaciones corresponden a investigaciones realizadas en ciudades de México y Chile con 7 y 5 registros respectivamente. En ciudades de Argentina y Bolivia se identificaron 3 investigaciones y en ciudades de Colombia, Uruguay y Cuba un estudio en cada país.

México es el país en el cual se han realizado más investigaciones que usan como marco teórico la Justicia Ambiental Urbana. La principal preocupación de las investigaciones son las diferencias socio ambiéntales presentes en la frontera entre México y Estados Unidos, identificando que en ciudades como Juárez y Tijuana hay una mayor exposición de la población a amenazas naturales (inundaciones y remoción) y antrópicas (emisiones químicas, contaminación del aire por industria), en comparación a ciudades como El Paso, localizada en Texas (Collins \& Grineski 2008; Collins, Grineski \& Romo 2009; McDonald \& Grineski 2011; Grineski et al 2012; Grineski ,Collins \& Romo 2015)

Chile es el segundo país de la región en términos de producción académica sobre Justicia Ambiental Urbana. Las investigaciones realizadas en ciudades chilenas se han concentrado en indagar como distintos grupos socioeconómicos son afectados de manera desigual por riesgos ambientales (Vásquez \& Salgado 2009; Romero, Fuentes \& Smith 2010), 
contaminación del aire (Romero, et al, 2013), temperatura e islas de calor (Molina, Romero \& Sarricolea, 2007) e impactos ambientales de la expansión urbana (Henriquez et al., 2009). También ha existido, aunque en menor medida, una preocupación por el acceso inequitativo a los espacios verdes urbanos (Vásquez y Salgado, 2009)

En Argentina se han realizado investigaciones sobre la exposición a agrotóxicos en zonas urbanas (Berger, 2009), el acceso desigual a servicios públicos (García 2003) y la distribución de indicadores de calidad ambiental entre distintas ciudades (Calemin, 2012). En Bolivia se han realizado dos investigaciones sobre el acceso desigual al agua en un ambiente urbano (Bustamante, 2012; Crespo, 2009). En Uruguay se ha realizado una investigación sobre los efectos de la contaminación del plomo en la ciudad (Renfrew, 2015), en Cuba una investigación sobre las acciones comunitarias para mejorar el medio ambiente urbano (Anguelovsky, 2013) y en Colombia sobre la contaminación del aire (Romero, et al 2013).

Es necesario resaltar que en la publicación de Romero-Lankao et al (2013) se hace un estudio comparativo de justicia ambiental urbana con respecto a la calidad del aire en Bogotá (Colombia), Santiago (Chile) y Ciudad de México (México) y en la investigación de Anguelovski (2013) se estudian casos de colectivos que buscan intervenir su entorno barrial en la Habana (Cuba) y Barcelona (España). Por esta razón las frecuencias absolutas son mayores a la cantidad total de artículos. Igualmente es necesario aclarar que las publicaciones de carácter conceptual no se tienen en cuenta para este cálculo.

\section{Fig. $N^{\circ}$ 2. Gráfico País en el que se investiga en las publicaciones}




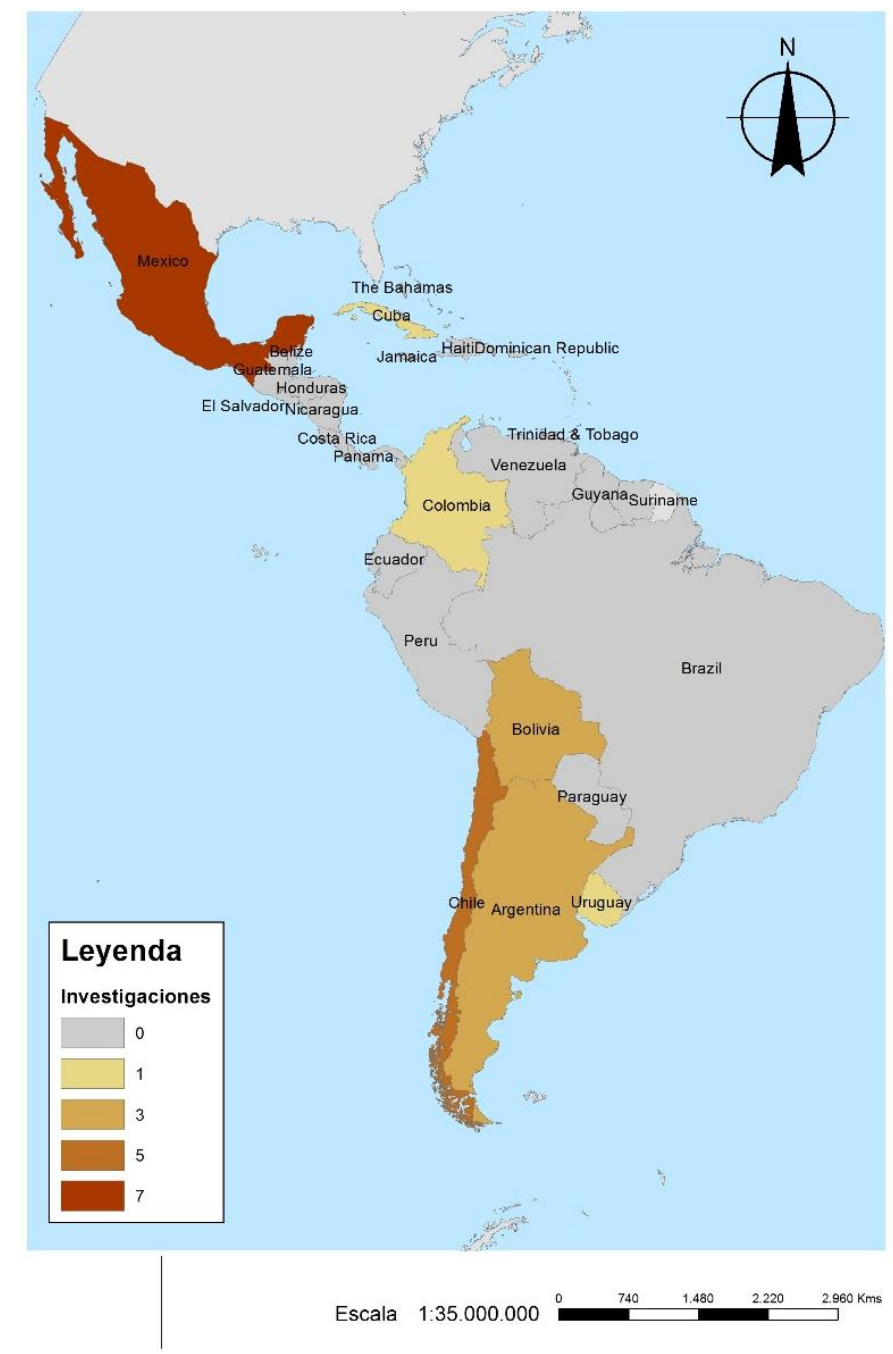

Fuente: Base de datos Scopus Redalyc y Scielo, Enero de 2017. Elaboración propia.

\section{DIMENSIONES INVESTIGADAS}

\section{Dimensión distributiva de la Justicia Ambiental Urbana}

La dimensión más investigada de la Justicia Ambiental Urbana es la distributiva, con 14 publicaciones, la cual evalúa la distribución social de bienes y males ambientales, ya sean amenidades urbanas, factores que generan contaminación ambiental o riesgos ambientales. De tal forma, en estos artículos se tiende a equiparar una distribución inequitativa con injusticia y se busca establecer si en un territorio específico se presenta una condición de injusticia ambiental.

En las investigaciones que se hacen sobre la dimensión distributiva de la justicia ambiental predomina un enfoque metodológico cuantitativo, principalmente en las 
investigaciones hechas en México (realizadas en Texas University en Estados Unidos), y en Chile (realizadas por geógrafos de la Universidad de Chile). En este tipo de estudios se han definido situaciones de injusticia a partir del reconocimiento de una afectación desigual (en términos estadísticos) de un grupo socioeconómicamente vulnerable por la contaminación. En general, son investigaciones que buscan generar conclusiones sobre la influencia que tienen las variables socioeconómicas en las variables ambientales, para así establecer escenarios de injusticia ambiental.

Es necesario resaltar que la totalidad de estos estudios cuantitativos basan sus hallazgos en el manejo de información secundaria, dado que realizan estimaciones a partir de información que no es recolectada directamente en campo. Generalmente las investigaciones hacen uso de Censos o padrones de carácter público para dar cuenta de las características sociales, que son evaluadas en función de variables ambientales derivadas de imágenes satelitales e inventarios municipales entre otros.

Con estas bases se construyen indicadores del nivel socioeconómico de la población que se contrastan con indicadores ambientales con tres tipos de procedimientos estadísticos. El primero, trata de describir los indicadores de carácter ambiental a través de diferencias de promedios o de valores absolutos entre grupos sociales según su nivel socioeconómico (García 2003; Vásquez \& Salgado 2009; Henríquez et al 2009; Collins, Grineski \& Romo 2009; Grineski, et al 2012; Norman et al 2012). El segundo tipo de procedimiento busca identificar correlaciones entre los indicadores ambientales y los socioeconómicos (Soltero, et al, 2015). Por último, en el tercer tipo de procedimiento busca predecir el nivel de la afectación ambiental con el nivel socioeconmico a partir de regresiones lineales o modelos generalizados (Molina, Romero \& Sarricolea 2007; Collins \& Grineski 2008; Romero, Fuentes \& Smith 2010; McDonald \& Grineski 2011; Celemín 2011; Romero et al 2013; Grineski, Collins \& Romo 2015)

En cuanto a los principales hallazgos, se puede mencionar que en México los resultados revelan situaciones de injusticia ambiental en las ciudades fronterizas. Por un lado, se destacan los resultados que muestran los escenarios de injusticia ambiental en ciudad Juárez respecto al Paso Texas, dado que se revelan claras disparidades espaciales del riesgo con base a la asignación diferencial de los factores de riesgo biofísico y social. (Collins \& Grineski 2009). Se señala que por cada hogar en el Paso que no cuenta con acueducto, en ciudad Juárez hay 23 hogares que tienen la misma problemática, ilustrando un caso de injusticia ambiental entre las dos ciudades (Grineski \& McDonald 2011). Estos estudios concluyen que en Juárez se incrementa la probabilidad de conformación de una isla de calor urbana y el riesgo de 
inundaciones frente al Paso (Grineski et al 2012), y que en ciudad Juárez la población está mayormente expuesta a contaminación industrial por presencia de maquilas. En Juárez la población más vulnerable son los menores de 10 años, dado que la probabilidad de que se vean afectados por riesgos biofísicos es significativamente más alta(Collins \& Grineski, 2009). Por otro lado, en Tijuana se concluye que hay mayor exposición a contaminación entre las madres cabezas de familia y los niños menores de 12 años por proximidad a parques industriales (Grineski et al 2015), y en Sonora que las comunidades vulnerables tienen una menor capacidad de prevención de la erosión y del control de inundaciones (Norman et al 2012).

En Chile, las investigaciones han demostrado que en Santiago la población de más altos ingresos vive en áreas de mejor calidad ambiental en comparación con el resto de los habitantes de la ciudad, en términos de temperaturas atmosféricas menos cálidas (Romero et al 2007) y que existe una diferencia significativa en los riesgos ambientales a los que se ven expuestas las áreas habitadas por población de grupos socioeconómicos diferentes (Vásquez \& Salgado 2009; Romero et al 2010)

En Argentina la investigación realizada en Buenos Aíres verifica un patrón tradicional de segregación residencial socioeconómica y su estrecha vinculación con la calidad ambiental medida en términos de un índice compuesto que relaciona recursos disponibles y problemáticas (Celemin 2009). En Tandil, la investigación muestra como la desigualdad social acentúa las desigualdades por riesgo de contaminación por ingesta de aguas contaminadas (García 2003).

\section{Dimensión participativa y política de la justicia ambiental urbana}

Aunque con menor frecuencia, en la región también se han desarrollado cinco investigaciones de carácter cualitativo para indagar en problemas de justicia ambiental derivados de la relación entre la sociedad civil y el estado en la toma de decisiones. De tal forma, se plantea que existe una condición de injusticia cuando se identifica un impacto desigual sobre diferentes grupos sociales derivado de una decisión política o por una participación desequilibrada en los procedimientos para tomar dichas decisiones.

La identificación de tales desigualdades se hace normalmente a partir de la reconstrucción de un conflicto social, en el cual un grupo social se declara afectado en su medio ambiente por una decisión o se declara afectado por la no participación en dicha decisión. Estas investigaciones se centran en la reconstrucción discursiva de los grupos sociales y los conflictos 
en los que se ven envueltos para mejorar su situación ambiental. Debido a lo anterior la metodología incluye la realización de entrevistas para dar cuenta del discurso de los grupos sociales sometidos a una condición injusta en la ciudad con respecto a su medio ambiente.

En los cinco estudios se llevaron a cabo entrevistas estructuradas para dar cuenta del discurso de los grupos sociales sometidos a una condición injusta en la ciudad con respecto al medio ambiente. Como complemento, en las investigaciones de Moore (2009) y Berger (2009) además de las entrevistas, se realizó un análisis documental para mejorar la comprensión del fenómeno.

Estos estudios giran principalmente entorno a los conflictos en los que están inmersos algunos grupos vulnerables con el estado en las diferentes ciudades estudiadas, concluyendo que esto se debe principalmente a la ausencia de políticas públicas claras para mantener o mejorar las condiciones medioambientales, ya sea por medio del suministro de recursos (como el agua) o de la gestión de impactos. De tal forma, en estas investigaciones se releva el papel de los movimientos sociales que entran en conflicto con el estado sobre la trayectoria de los mismos conflictos.

En el caso de Oaxaca- México, Moore (2009) da cuenta del conflicto social provocado por la gestión de basuras y la afectación de comunidades vulnerables que concentran los impactos negativos derivados. En la ciudad de Córdoba - Argentina, Berger (2009) investiga sobre la auto organización ciudadana para la defensa de la salud ambiental por la exposición a agrotóxicos y se concluye que en el contexto del estado democrático de derecho, son las prácticas ciudadanas las que defienden lo público de la política a través de su acción, y no las estructuras institucionales del estado, supuestas garantes de los derechos.

En Cochabamba - Bolivia, Bustamante (2012) da cuenta de cómo las organizaciones sociales están luchando para defender su identidad rural en condiciones críticas por los altos niveles de contaminación generados por la planta de tratamiento de aguas residuales. En otra investigación, Mehta (2009) da cuenta de los colectivos sociales que buscan la protección del recurso hídrico resaltando que las políticas públicas al respecto no han podido hacer frente a las necesidades específicas de determinadas localidades que se mantienen excluidas.

En la Paz - Bolivia, Crespo (2009) analiza las formas de organización social que buscan garantizar el acceso equitativo al agua, llegando a la conclusión de hay dos tipos de racismo ambiental, uno por los impactos que generan las actividades económicas que son asumidos por las poblaciones indígenas y dos por la reducción en el acceso y uso de recursos naturales que tienen estos grupos. 
En Montevideo - Uruguay se analiza el discurso comunitario en torno a la contaminación de plomo, mostrando como las respuestas desde los grupos de base reflejan la emergencia del ambiente como categoría jurídica, de acción y de pensamiento, y de la justicia ambiental como método y forma de lucha política contra las injusticias ecológicas y sociales (Renfrew, 2015).

Mención aparte requiere la investigación de Isabelle Anguelovski (2013) desarrollada en la Habana y Barcelona, la cual se centra directamente en la acción de algunos colectivos que tiene como objetivo mejorar las condiciones ambientales de su entorno urbano, al margen de la acción del estado.

\section{Publicaciones de carácter conceptual}

Las tres publicaciones de carácter conceptual tienen en común el desarrollar una reflexión en torno al riesgo y la manera de vincularlo a la gestión urbana para evitar o prevenir condiciones injustas (Caballero 2013; Campos et al 2015).

En primer lugar, en la publicación de Caballero (2013) se plantea una discusión sobre la importancia de la inclusión de la evaluación de los riesgos de origen antrópico y los derivados de amenazas naturales en las prácticas de planificación urbana. La discusión planteada, busca posicionar la gestión del riesgo como un mecanismo que permitiría ampliar el sentido del derecho a la ciudad, e incluir el acceso equitativo a bienes y servicios urbanos dignos y recuperar la noción de justicia frente a una vulnerabilidad que ha sido construida socialmente. De tal forma, en el fondo se identifica a la gestión del riesgo y a la planificación urbana como unos mecanismos para producir o eliminar condiciones injustas respecto al medio ambiente.

La publicación realizada por Campos et al (2015) discute las nociones de vulnerabilidad y riesgo y de justicia ambiental y justicia espacial, en el contexto de los riesgos naturales y los desastres. Se afirma que la vulnerabilidad y el riesgo son construcciones sociales, derivadas de las transformaciones antrópicas del ambiente y que, por lo tanto, las consecuencias se distribuyen de manera inequitativa entre distintos grupos como reflejo de las desigualdades sociales existentes. A partir de esta vinculación conceptual entre riesgo e inequidad se plantea que la justicia ambiental y la justicia espacial pueden ser conceptos guías mediante los cuales se pueden minimizar las inequidades y los impactos de los desastres.

La revisión conceptual que hacen Anguelovski \& Martinez Alier (2014) contiene un repaso de los conceptos que utilizan los movimientos ambientalistas en su activismo social. Son 
revisados conceptos tales como deuda ecológica, la justicia ambiental, pasivos ambientales, el acaparamiento de tierras, la gentrificación del medio ambiente, la responsabilidad corporativa, la justicia climática y la soberanía alimentaria como claves de las redes de la justicia mundial del medio ambiente. Además, se plantea en la discusión que la investigación comparativa puede ayudar a desentrañar el uso de lenguajes de valoración diferentes, de manera tal que se puedan plantear alternativas al desarrollo económico en su versión hegemónica.

\section{CONCLUSIONES Y DISCUSION}

La revisión del estado de la investigación en Justicia Ambiental Urbana en Latinoamérica indica que se trata de un tema novedoso sobre el cual no existe gran volumen de publicaciones. Por tanto, en la región se ha hecho un acercamiento parcial a un concepto que resulta complejo y multidimensional.

A la luz de las dimensiones de análisis de la justicia ambiental urbana, se puede afirmar que la investigación en Justicia Ambiental en las ciudades de la región (1) ha estado importantemente concentrada en la dimensión distributiva, especialmente de los males ambientales, cuestión que concuerda con la tendencia mundial descrita por Walker (2009), y (2) ha abordado de manera separada cada una de las dimensiones sin presentarse aún un debate sobre una lectura integral del fenómeno. Esto no parece acompañar adecuadamente los intereses de los movimientos sociales y políticos que mueven la agenda de justicia ambiental en Latinoamérica (Vásquez et al 2017)

Se concluye que la investigación ha estado centrada en la conceptualización de la Justicia Ambiental Urbana como un problema de equidad e inequidad, por lo cual ha predominado la aplicación de metodologías de carácter cuantitativo, a partir de las cuales se busca poner de manifiesto y de manera objetiva la situación de desigualdad que tienen los grupos sociales con respecto a la calidad de su medio ambiente en la ciudad. De tal forma, la investigación se ha centrado en la identificación y descripción cuantitativa del fenómeno distributivo, más que en su comprensión discursiva y despliegues de poder. En estos estudios, se identifica y describe la condición de justicia o injusticia ambiental a partir de la construcción de indicadores de nivel socioeconómico, sobre la base de datos de ingreso, nivel de educación y tipo de ocupación, principalmente. Por lo cual no han sido incorporados en los estudios problemas raciales, étnicos, religiosos o migratorios. 
Con respecto a las investigaciones de carácter cualitativo sobre la dimensión política, se puede concluir que en tanto describen el conflicto de algunos movimientos sociales con respecto a las condiciones negativas de su medio ambiente urbano, el estado y sus procedimientos de reglamentación o ejecución de políticas, aparece como protagonista principal de los discursos que se reconstruyen con dichos grupos. En la medida de que se trata de investigaciones que buscan poner de manifiesto las formas de gestión del conflicto por parte de los grupos sociales, el estado aparece como la contraparte que por acción u omisión contribuye a sostener las condiciones injustas en el ambiente que los afectan. Para ello, la recolección de discursos a través de entrevistas estructuradas, se constituye en la principal técnica de investigación que permite validar los resultados.

En general, los resultados arrojados por la revisión ponen manifiesto que la Justicia Ambiental Urbana es un terreno en el cual aún se pueden hacer aportes sustanciales a partir de investigaciones provenientes de diferentes contextos, diferentes perspectivas metodológicas y diferentes fuentes de información. Teniendo en cuenta la baja cantidad de investigaciones que se presentan en Latinoamérica, se hace necesario iniciar debates conceptuales y metodológicos sobre la situación ambiental en las ciudades de la región, de manera tal que surjan nuevas preguntas de investigación que enriquezcan la producción científica sobre Justicia Ambiental Urbana.

Lo anterior resalta la necesidad de que en la investigación sobre Justicia Ambiental Urbana se reflexione sobre la combinación de estrategias metodológicas, más allá de consideraciones epistemológica, ya que teniendo en cuenta las dimensiones del concepto en estudio esto debe ser explícitamente deseable. Es necesario recordar que las ciencias sociales en la actualidad buscan demostrar que los principios subyacentes a la generación de conocimiento científico son válidos tanto para investigación cuantitativa como para la cualitativa (Rivadulla 2012).

En otra dirección, es necesario tener en cuenta que la presente revisión únicamente utilizó bases de datos en las que se encuentran las investigaciones publicadas en revistas formalmente indexadas. Por tanto, quedaron por fuera de la revisión un número indeterminado de investigaciones que se pueden haber realizado como proyectos académicos y trabajos de grado, cuyos resultados no han sido publicados o no han sido publicados en las revistas que componen las bases de datos utilizadas en esta revisión.

Igualmente, hay que tener en cuenta que en la presente revisión solo se incluyen aquellos artículos que explícitamente hacen referencia al termino justicia ambiental, lo que 
puede significar que otros trabajos donde se analizan problemas de justicia ambiental pero usando aproximaciones teóricas diferentes - e.g. ecología política - no fueron considerados. Por ejemplo, ampliar el panorama de búsqueda incluyendo conceptos como "conflictos ambientales urbanos", podría arrojar una mayor cantidad de artículos publicados, especialmente de carácter cualitativo, teniendo en cuenta que es el enfoque principal de la ecología política (Anguelovski y Martinez Alier ,2014).

\section{REFERENCIAS}

ANGUELOVSKI, Isabelle. 2013. "New Directions in Urban Environmental Justice: Rebuilding Community, Addressing Trauma, and Remaking Place". Journal of Planning Education and Research, 33: 160-175. DOI: 10.1177/0739456X13478019.

ANGUELOVSKI, Isabelle \& Martines, Alier. 2014. "The 'Environmentalism of the Poor' revisited: Territory and place in disconnected glocal struggles". Ecological Economics, 102:167-176. http://dx.doi.org/10.1016/j.ecolecon.2014.04.005.

BERGER, Mauricio y Ortega, Francisco. 2009. "Poblaciones expuestas a agrotóxicos: autoorganización ciudadana en la defensa de la vida y la salud, Ciudad de Córdoba, Argentina". Physis, 20 (1): 119-143.

BUSTAMENTE, Rocio \& Aurore Medieu. 2012. "Struggling at the Borders of the City: Environmental Justice and Water Access in the Southern Zone of Cochabamba, Bolivia". Environmental Justice, 5 (2): 89-92. DOI: 10.1089/env.2011.0018

CABALLERO, Zeitún, \& Elsa Lilly. 2013. "Los riesgos urbanos y la justicia urbana en Centroamérica". Anuario de Estudios Centroamericanos, 39.

CAMPOS, Milagros, Alejandra Toscana-Aparicio, \& Juan Campos Alanís. 2015. "Riesgos socionaturales: vulnerabilidad socioeconómica, justicia ambiental y justicia espacial". Cuadernos de Geografía - Revista Colombiana de Geografía, 24 (2): 53-69. DOI: dx.doi.org/10,15446/rcdg.v24n2.50207

CELEMIN Juan. 2012. "Calidad ambiental y nivel socioeconómico: su articulación en la región metropolitana de Buenos Aires". Scripta Nova, 441.

CHAKRABORTY Jayajit, \&Green Donna .2014. Australia's first national level quantitative environmental justice assessment of industrial air pollution. Environ. Res. Lett. 9 (2014). DOI: 10.1088/1748-9326/9/4/044010

COLLINS Timothy W \& Grineski Sara E. 2008. "Exploring patterns of environmental injustice in the Global South: Maquiladoras in Ciudad Juarez, Mexico". Popul environ, 29: 247-270. DOI:10.1007/s11111-008-0071-z 
CUTTER, Susan. 1995. "Race, class and environmental justice". Progress in Human Geography, 19 (1): 111-122. DOI: 10.1177/030913259501900111.

DALE Laura, Goudreau Sophie, Perron Stephane, Ragettli Martina, Hatzopoulou Marianne \& Smargiassi Audrey. 2015. Socioeconomic status and environmental noise exposure in Montreal, Canada. BMC Public Health. 15 (205) DOI: 10.1186/s12889-015-1571-2.

ENVIRONMENTAL JUSTICE ATLAS, 2016. EJAtlas | Mapping Environmental Justice. https://ejatlas.org/.

GARCÍA María. 2003. "El desigual acceso a servicios públicos urbanos. Brechas sociales y riesgo ambiental en el caso de Tandil, Argentina". Disertación Centro de Investigaciones Geográficas (CIG), Fac. Cs. Humanas, Universidad Nacional del Centro de la Provincia de Buenos Aires.

GELOBTER, Michel. 1994. "The meaning of urban environmental justice". Fortham Urban Law Journal, 21 (3): 841-856.

GERMANI Ana, Morone Piergiuseppe \& Testa Giuseppina. 2014. Environmental justice and air pollution: a case study on Italian provinces. Ecological Economics. 106: 69-82. DOI: 10.1016/j.ecolecon.2014.07.010

GRINESKI, Sara, Timothy Collins, \& María Romo. 2015. "Environmental injustice along the USMexico border: residential proximity to industrial parks in Tijuana", Mexico. Environ. Res. Lett, 10: 1-10. DOI: 10.1088/1748-9326/10/9/095012.

2009. "Vulnerability to environmental hazards in the Ciudad Juarez (Mexico)-El Paso (USA) metropolis: A model for spatial risk assessment in transnational context". Applied Geography, 29 (3): 448-461. DOI: 10.1016/j.apgeog.2008.10.005.

GRINESKI, Sara, Timothy Collins, María Romo, Paula Ford, Raed Aldouri, Gilberto Velázquez, \& Duanjun Lu. 2012. "Climate change and environmental injustice in a bi-national context". Applied Geography, 33: 25-35. DOI: 10.1016/j.apgeog.2011.05.013.

HOLIFIELD, R., Porter, M., \& Walker, G. (2010). Spaces of Environmental Justice-Frameworks for Critical Engagement. Spaces of environmental justice, 1-22.

HARVEY, David. 1996. Justice, nature and the geography of difference. Cambridge - Blackwell Publishers

HENRÍQUEZ Cristian, Arenas Federico, Romero Hugo, \& Azócar Gerardo 2009. Justicia socioambiental y sostenibilidad en el crecimiento de las ciudades medias de Chillán y Los Ángeles (Chile). En Las Ciudades Medias o Intermedias en un mundo globalizado. Editado por la Universitat de Lleida, 389-411, Lleida Càtedra UNESCO.

JIMÉNEZ Juan. 2015. Desigualdad, concentración del ingreso y tributación sobre las altas rentas en América Latina. En Libros de la CEPAL, № 134. Editado por la CEPAL, Santiago de Chile, Comisión Económica para América Latina y el Caribe (CEPAL).

JONES Miranda, Diez-Roux Ana, Hajat Anjum, Kershaw Kiarri, O'Neill Marie, Guallar Eliseo, Post Wendy, Kaufman Joel, Navas Ana. 2014. Race/ethnicity, residential segregation, and exposure to 
ambient air pollution: the multi-ethnic study of atherosclerosis (MESA). Public Health, 104 (11): 2130-7 DOI: 10.2105/AJPH.2014.302135.

KHAN, K. S., Kunz, R., Kleijnen, J., \& Antes, G. (2003). Five steps to conducting a systematic review. Journal of the Royal Society of Medicine, 96(3), 118-121.

LAURIAN Lucie and Funderburg Richard. 2014 Environmental justice in France? a spatiotemporal analysis of incinerator location. Journal of Environmental Planning and Managemen. 57: 424-46. DOI: 10.1080/09640568.2012.749395

MCDONALD, Yolanda y Sara Grineski. 2011. "Disparities in access to residential plumbing: a binational comparison of environmental injustice in El Paso and Ciudad Juarez". Popul Environ, 34: 194-216. DOI: 10.1007/s11111-011-0154-0.

MEHTA, Lyla, Jermey Allouche, Alan Nicol y Anna Walnycki. 2013. "Global environmental justice and the right to water: The case of peri-urban Cochabamba and Delhi". Geoforum, 54: 158-166. DOI: 10.1016/j.geoforum.2013.05.014.

MOHAl Paul, Pellow David, Roberts Timmons. 2009. Environmental justice. Annual Review of Environment and Resources. 34 (1): 405-30. DOI: 10.1146/annurev-environ-082508-094348

MOLINA Melandra, Romero Hugo, Sarricolea Pablo. 2007. "Características socio ambientales de la expansión urbana de las Áreas metropolitanas de Santiago y Valparaíso". En Chile: Del país urbano al metropolitano. Editado por Geolibros, 187 - 200, Santiago: Pontificia Universidad Católica.

MOORE, Sarah. 2009. "The Excess of Modernity: Garbage Politics in Oaxaca, México". The Professional Geographer, 61 (4): 426-437. DOI: 10.1080/00330120903143375.

NOGUEIRA Helena, Gama Augusta, Mourao Isabel, Marques Vitor, Ferrao Maria and Padez Crstina. 2013. The associations of SES, obesity, sport activity, and perceived neighborhood environments: is there a model of environmental injustice penalizing Portuguese children?. American Journal of Human Biology. 25: 434-6. DOI: 10.1002/ajhb.22384

NORMAN, Laura, Miguel Villareal, Francisco Lara, Yongping Yuan, Wenming Nie, Sylvia Wilson, Gladyz Amaya, y Rachel Sleeter. 2012. "Mapping socio-environmentally vulnerable populations access and exposure to ecosystem services at the U.S. -Mexico borderlands". Applied Geography, 34: 413-424. DOI: 10.1016/j.apgeog.2012.01.006.

RENFREW, D 2007. 'Justicia ambiental y contaminación por plomo en Uruguay'. En antropología social y cultural en Uruguay, Unesco, Montevideo.

RIVADULLA, María José. (2012). "Métodos combinados: una aplicación al estudio de la ciudad informal". En Revista de Estudios Sociales, Universidad Católica de Uruguay, 3 (3): 127-150.

ROMERO Hugo, Fuentes Claudio \& Smith Pamela. 2010. "Ecología política de los riesgos naturales y de la contaminación ambiental en Santiago de Chile: necesidad de justicia ambiental". Scripta Nova, 331 
ROMERO, Patricia 2007, 'Are we missing the point? Particularities of urbanization, sustainability and carbon emissions in Latin American cities'. Environment and Urbanization, 83, (1): 159-175. DOI: 10.1177/0956247807076915

ROMERO, Patricia, Hua Qin, \& Mercy Borbor. 2013. "Exploration of health risks related to air pollution and temperature in three Latin American cities". Social Science \& Medicine, 83: 110118. http://dx.doi.org/10.1016/j.socscimed.2013.01.009.

SAROKIN, David, y Jay schulkin. 1994. "Environmental justice: co-evolution of environmental concerns and social justice". The Environmentalist, 14, (2): 121-129. DOI: 10.1007/BF01901305.

SOLTERO Erica, Scherezade Mama, Pacheco \& Lee Rebbeca. 2015. "Physical Activity Resource and User Characteristics in Puerto Vallarta, Mexico". RETOS. Nuevas Tendencias en Educación Física, Deporte y Recreación, 28: 203-206.

UNITED NATIONS. 2014. World Urbanization Prospects 2014: Highlights. United Nations Publications.

VÁSQUEZ Alexis, \& Marcela Salgado. 2009. "Desigualdades socioeconómicas y distribución inequitativa de los riesgos ambientales en las comunas de Peñalolén y San Pedro de la Paz". Una perspectiva de justicia ambiental. Revista de Geografía Norte Grande, 43: 95-110. DOI: 10.4067/S0718-34022009000200006.

VÁSQUEZ, Alexis.; Lukas, Michael; Salgado, Marcela \& Mayorga, José. 2017. "Urban environmental (in)justice in Latin America: the case of Chile". Capitol de libro en: Gordon Walker \& Ryan Holifield. Handbook of Environmental Justice. Taylor \& Francis Books. En prensas.

WALKER J. 2009. Environmental justice and normative thinking. Antipode. 41(1):203-205 ZOU BIN, Peng Fen, Wan Neng, Wilson Gaines, Ying Xiong Y. 2014. Sulfur dioxide exposure and environmental justice: a multi-scale and source-specific perspective Atmos. Pollut. 5(3): 491-9 DOI:10.5094/APR.2014.05

Trabalho enviado em 13 de junho de 2017.

Aceito em 17 de julho de 2017. 